\title{
Occlusal seduction
}

One of the longstanding debates in dentistry is the role of occlusion in temporomandibular disorders (TMD) and this issue sees the publication of an interesting research paper that adds some useful information into the debate. The paper is by Wassell, Adams and Kelly (page 35) and looks at the effect of using stabilising splints in the initial treatment phase, but unlike many studies it does this in general practice rather than a hospital setting.

The findings will certainly stimulate debate, providing ammunition for both those who feel that most occlusal treatment in TMD is either unnecessary or irrelevant, and those who believe that treating the occlusion is a vital part of TMD therapy. However, the really useful point of the research is that it helps provide an evidence-based approach to TMD therapy which is rare in itself, although obviously one paper in such a complex clinical situation could hardly be expected to be the panacea.

The study looked at 72 patients attending nine general dental practitioners (GDPs) who were randomly provided with either a stabilising splint with occlusal coverage or a similar splint without any occlusal coverage (controls). Importantly, the patients with the control splint were given a splint with occlusal coverage if it was felt that they were not responding to treatment enough, and 17 patients did cross over from a control splint to an occlusal-coverage splint, but the remainder of the control group (34 in total, so 17 who did not cross over) continued with their control splint to the end of the study (six weeks after the start).

The findings were that all patients benefited from the treatment and showed improvement in clinical signs and symptoms, regardless of whether they wore a stabilising splint with occlusal coverage or a control splint without. The researchers concluded that there were no statistical differences in outcome, regardless of the splint worn.

Now, before the non-occlusion devotees leap to the conclusion that TMD has nothing to do with the occlusion I should point out that six of the patients did have further occlusal adjustment as prescribed by both the dentist and the consultant, indicated by either the return of pain following splint removal or the patient stating that their occlusion was uncomfortable. However that leaves 66 patients who apparently did not require any occlusal adjustment following splint therapy, which enabled the researchers to state what was, for me, the key sentence in their paper: 'These findings imply that four out of five TMD patients can be managed by knowledgeable GDPs using reversible treatment. Brave words indeed. And the key word in that key sentence (again for me) is 'knowledgeable'.

The GDPs in the trial had received training in occlusion and were members of an occlusal study group, and were considered by the dental hospital consultant to be well qualified to diagnose and treat TMD. This is the real difficulty in TMD matters, as there are a number of 'weekend' courses on occlusion (some are only one day) where GDPs can attend and then theoretically presume themselves to be equally 'well-qualified' to launch into TMD treatment. This is not so worrying if these GDPs confine themselves to reversible therapy, but my experience is that often the treatment is irreversible, involving the entire range of therapies from simple occlusal adjustment to full mouth rehabilitation, all in the name of treating TMD. Some people go even further, treating headaches and other medical symptoms which, it is claimed, respond to occlusal therapy.

I know the seductive attraction of occlusal therapy because I have travelled the road myself in my clinical days. My experience at dental school was that no-one seemed to know much about occlusion, and in my naivety I assumed this was wrong and that there should be an answer. When so-called 'occlusal gurus' appeared to know what the answer was I eagerly listened and initially believed. Luckily (for my patients) I was never a fan of the restorative solution and quickly discovered that occlusion was not only a complex subject but that the people at my dental school had indeed been right after all - there was no sure answer to the multifactorial condition we now call TMD.

However, as we progress in knowledge we are now beginning to get a glimpse of a possible answer that is far more evidence-based than in the past, and the paper by Wassell et al may not be the whole truth and nothing but, but it is indeed a small step in the right direction.

Mike Grace, Editor m.grace@bda.org doi: 10.1038/sj.bdj.4811409 